\title{
Update of Hyperbaric Oxygen Therapy in the Management of Chronic Wound
}

\section{Ibrahim A Albrethen ${ }^{\star, \dagger}, 1$, Turki M Alshehri ${ }^{2}$, Khalid A Albraithen ${ }^{3}$, Abdullah I Alenezi ${ }^{4}$, Hussein M Alkahtani ${ }^{5}$, Ahmad Y Albalawi ${ }^{6}$}

\author{
${ }^{1}$ Aeromedical center,Dhahran, Saudi Arabia. \\ ${ }^{2}$ Alhada Military Hospital, Taif, Saudi Arabia \\ ${ }^{3}$ PSMMC, Riyadh, Saudi Arabia \\ ${ }^{4}$ Dhahran Military Hospital, Dhahran, Saudi Arabia \\ ${ }^{5}$ Jubail Naval Military Hospital, Jubail, Saudi Arabia \\ ${ }^{6}$ King Salman Military Hospital, Tabuk, Saudi Arabia
}

(DDOI: https://doi.org/10.15520/jcmro.v3i02.255

Accepted 01-02-2020; Received 10-01-2020; Publish Online 02-02-2020

\author{
Reviewed By: \\ Dr. K. Daniel \\ Department: \\ Reviewer/CMRO
}

\begin{abstract}
Hyperbaric oxygen therapy (HBOT) is a type of therapy for wound management that employs pressurized oxygen, which patients breathe for a set period. The aim is to increase the amount of oxygen in a person's body, which should aid the process of wound healing, which is especially crucial for chronic wounds. Multiple evidence from clinical trials and studies suggest that HBOT is more effective when compared to traditional therapies in treating chronic wounds, and especially when managing wounds that develop as a result of diabetes. Additionally, two studies conducted on rats with diabetes and patients with foot ulcers suggest that HBOT can address these complex cases, which can aid in reducing the amputation risk. This paper reviews evidence, which proves that HBOT is an effective chronic wound management strategy.
\end{abstract}

\section{HBOT:}

HBOT involves placing a patient in a room with pressurized oxygen, where the person can breathe it through a tube. Complex cases with wounds that do not heal for over three months present a danger to patients. In some cases, the latter may require amputation to avoid the further risk associated with their non-healing wound, and this risk can be addressed by employing HBOT. This research paper aims to examine scholarly evidence that explores the topic of HBOT use as a way of chronic wound management.

\section{THE EFFECT ON THE MANAGEMENT OF CHRONIC WOUND:}

Hyperbaric oxygen therapy is a treatment option that takes place in a specialized room. In this facility, a patient is exposed to pure oxygen. This paragraph aims to examine scholarly articles that provide empirical evidence sup-

\footnotetext{
* Corresponding author

† Email: editor@cmro.in
}

porting the use of HBOT in the management of chronic wounds to determine whether it is more effective when compared to other treatment options. Firstly, according to Lam, Fontaine, Ross, and Chiu (2017) [1] , it is the "the administration of $100 \%$ oxygen at pressures greater than 1.4 atmospheres absolute in a series of treatments, can be used as adjunctive therapy in many wound care settings" (p. 181). The primary effect of this therapy is a decrease of inflammation and improvement of oxidation and neovascularization, all of which help wounds heal better. Lam et al. (2017) [2] and Kim et al. (2018) [3] argue that despite the benefits of this approach, many medical professionals are unfamiliar with HBO, with explains the limited use of this approach in a clinical setting, despite the fact that it significantly decreases the risk of amputation. Thus, HBOT implies using oxygen under predefined pressure to improve the amount of oxygen in a person's body, which is associated with better wound healing, since the process requires O2. Figure 1 presents the critical stages of wound healing and demonstrates the effect of HBOT, explaining the dependency of wound healing on oxygen.

Chronic wounds are conditions that are characterized by a lack of proper healing process. This definition means that 


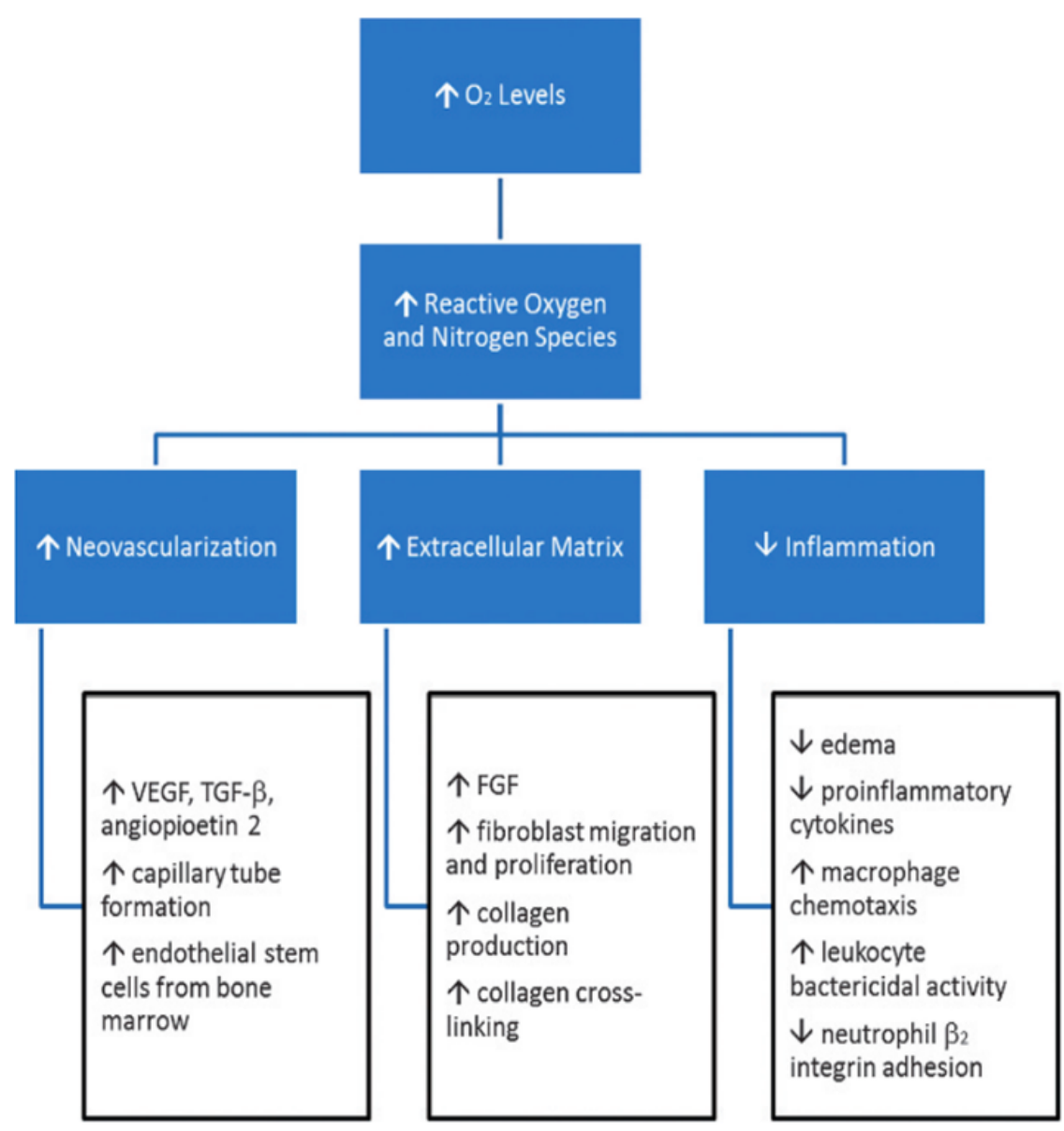

Figure 1. HBOT mechanism in wound healing (Lam et al., 2017).

a person's injury does not go through the typical stages in a set period, as suggested by the specifics of this wound (Lam et al., 2017) [2]. In general, wounds that do not go through this process within three months are considered to be chronic, and they require additional attention from a healthcare professional. Huang, Heyboer, and Savaser (2019) [4] explore the role of oxygen in the process of wound healing since this is the central aspect of HBO. According to the authors' research, it is involved in every stage of wound healing, such as "fibroblast replication, collagen deposition, 1 angiogenesis, $2-5$ resistance to infection, $6-8$ and intracellular leukocyte bacterial killing" (Huang et al., 2019, p. 27) [4] . However, apart from theoretic evidence, it is necessary to explore studies that test this theory about HBOT's effect on chronic wounds. In the study by Sureda et al. [5] (2016), patients with chronic wounds, which lasted on average for more than twenty months, experience an improvement after one month of HBOT. Mainly, the decrease in wound size and improvement of the healing rate were reported.

In some cases, however, HBOT cannot be used as a single therapy and can be effective only if applied as an additional measure. Huang (2018) [6] states that despite the benefits of HBO, some patients may be not susceptible to this treat- ment, regardless of the mentioned benefits, resulting in a wound's failure to heal. This is applicable to patients with diabetes who have wounds on their lower extremities. Additioanlly, Fedorko et al. (2016) [7] explored the effect of HBOT on patients with signs for amputation. Out of the two groups, one assigned to HBOT and the other to traditional therapy, approximately $20 \%$ experienced improvement. This suggests that for severe cases where amputation is recommended, HBOT does not increase the chances for healing.

Alternatively, some evidence suggests that HBOT can be used to treat wounds of people with diabetes. Chen, $\mathrm{Wu}$, Hsu, Hsieh, and Chou (2017) [1] conducted a study focusing on how HBOT can be used to address foot ulcers, which are a common complication of diabetes. This condition is characterized by an open sore, usually on a patient's foot, and in some cases can damage not only one's skin but also bones and other structures of a person's body. In the experiment by Cheng et al. (2017) [1] , individuals with foot ulcers were divided into two groups - one received standard treatment therapy, and the other was engaged in a combination of standard treatment and HBOT. The results suggest that HBOT is more effective since $25 \%$ of individuals' wounds were in complete closure as opposed to $5 \%$ from the control 
group. The sessions lasted for two hours, five times a week for a month, and factors such as "inflammation index, blood flow, and health-related quality of life from pretreatment to 2 weeks after the last therapy ended" improved significantly in the group that was subjected to HBOT. This suggests that the therapy is indeed more effective when compared to traditional approaches.

Some researchers argue that the limited use of HBOT is a result of limited research on the subject and a lack of understanding in relation $t$ how the therapy actually works and what effect it has on a person's body. van Neck et al. $(2017)[7,8]$ conducted a study on rats, aiming to determine if HBOT treats wounds in subjects with diabetes. The focus of HBOT practitioners and researchers is often directed towards diabetes, since

\section{DISCUSSION:}

In general, evidence suggests that HBOT is an effective management strategy for chronic wounds. The main idea that guides the use of HBOT for wound treatment is the fact that a person's body needs oxygen in order to heal. HBOT can be used as an additional therapy to support the wound healing process of chronic injuries for patients with diabetes, which can reduce the risk of wound progression that will lead to an amputation. However, as was explained above, for patients who are already at risk of amputation, HBOT does not appear to provide additional benefits, especially of their lower limbs are injured.

The specifics of HBOT allow using it for treating wounds, more specifically those that do not heal naturally as a result of diabetes or due to other reasons. It is evident that chronic wounds are dangerous for patients because they can result in stress and can cause complications. HBO, however, also has several side effects that must be considered. For example, Fedorko et al. (2016) suggest that this therapy can result in myopia, the collapse of a lung, issues with the middle ear, or seizures. These are all consequences of pure oxygen's impact on a person's body, although another danger of its use is a possible fire hazard. Therefore, HBOT is an effective treatment for chronic wounds. However, it should be used with caution, and a patient's state should be monitored throughout and after a procedure.

\section{CONCLUSION:}

HBOT is a method that has been known to the medical and scientific community for a long time, however, its use is still limited. It employs and leverages the basis of natural wound healing, which requires oxygen at different stages. Some evidence suggests that HBOT effectively addresses this and reduces inflammation, allowing for better healing. Since chronic wounds usually do not heal after three months, HBOT can be used to address the main problems associated with the improper healing process. However, HBOT does not aid patients who are recommended an amputation.

\section{REFERENCES}

1. Chen, C. Y., Wu, R. W., Hsu, M. C., Hsieh, C. J., \& Chou, M. C. (2017). Adjunctive hyperbaric oxygen therapy for healing of chronic diabetic foot ulcers. Journal of Wound, Ostomy and Continence Nursing, 44(6), 536-545.

doi:10.1097/won.0000000000000374

2. Fedorko, L., Bowen, J., Jones, W., Oreopoulos, G., Goeree, R., Hopkins, R., \& O'Reilly, D. (2016). Hyperbaric oxygen therapy does not reduce indications for amputation in patients with diabetes with nonhealing ulcers of the lower limb: A prospective, double-blind, randomized controlled clinical trial. Diabetes Care, 39(3), 392-399. doi:10.2337/dc15-2001 3. Huang E. (2018). Comment on Santema et al. hyperbaric oxygen therapy in the treatment of ischemic lower-extremity ulcers in patients with diabetes: Results of the DAMO2CLES multicenter randomized clinical trial. Diabetes Care. 41, 112119. doi:10.2337/dc17-0654106.

4. Huang, E., Heyboer III, M., \& Savaser, D. J. (2019). Hyperbaric oxygen therapy for the management of chronic wounds: Patient selection and perspectives. Chronic Wound Care Management and Research, 6, 27-37. doi:10.2147/ cwcmr.s175721

5. Kim, D., Rao, A., Kaplan, S., Baksh, F., Caprioli, R., Haight, J., Oropallo, A. (2018). The use of indocyanine green fluorescence angiography to assess perfusion of chronic wounds undergoing hyperbaric oxygen therapy. Undersea Hyperb Medicine, 45(6), 663-671.

6. Lam, G., Fontaine, R., Ross, F. L., \& Chiu, E. S. (2017). Hyperbaric oxygen therapy. Advances in Skin \& Wound Care, 30(4), 181-190. doi:10.1097/01.asw.0000513089.75457.22 7. Sureda, A., Batle, J. M., Martorell, M., Capó, X., Tejada, S., Tur, J. A., \& Pons, A. (2016). Antioxidant response of chronic wounds to hyperbaric oxygen therapy. PloS One, 11(9), e0163371.

doi:10.1371/journal.pone.0163371

8.Van Neck, J. W., Tuk, B., Fijneman, E., Redeker, J. J., Talahatu, E. M., \& Tong, M. (2017). Hyperbaric oxygen therapy for wound healing in diabetic rats: Varying efficacy after a clinically-based protocol. PloS One, 12(5), e0177766. doi:10.1371/journal.pone.0177766 